\title{
Stomach Problems that Causes Gastric Pain from the Perspective of Iranian Traditional Medicine: A Mini Review
}

\author{
Roshanak Mokaberinejad and Elham Parsa* \\ Department of Traditional Medicine, Shahid Beheshti University of Medical Sciences, Iran
}

Submission: September 09, 2018; Published: October 17, 2018

*Corresponding author: Elham Parsa, Department of Traditional Medicine, School of Traditional Medicine, Shahid Beheshti University of Medical Sciences, Tehran, Iran; Email: Dr.eparsa@sbmu.ac.ir

\begin{abstract}
The stomach disorders are among the most common gastrointestinal diseases that reduce the quality of life. Traditional medicine had been important in treatment of disease since long ago. The causes of stomachache in Iranian traditional medicine are, abnormal humeric substance in the stomach, Yellow bile or Safra and Black bile or Sauda in the stomach, existence of gas in the stomach, excessive filling of the stomach, excessive sensation of the stomach. Diagnosis and treatment of stomach pain is important, gastrointestinal tract disorders spreads to other organs and causes significant problems.
\end{abstract}

Keywords: Stomach Gastric pain; Iranian Traditional Medicine

\section{Mini Review}

The stomach disorders are among the most common gastrointestinal diseases that reduce the quality of life and heavy economic burden, the prevalence of indigestion in Western countries is $18-30 \%$ and in Asian countries is $14 \%-8 \%$, the incidence of reflux in the world is $25 \%-30 \%$ and in Asia is $3 \%-7 \%$ [1].

The prevalence of gastric ulcer in the world is about $15 \%$ $-5 \%$, and it is estimated at $41 \%$ in a study conducted in Iran, which is rising with increasing age and men are more likely than women to develop the disease [2]. The stomach as a member of the muscle tissue [3], that plays a vital role in digestion of foods [4], whatever is eaten, and everything that it reaches the body first passes through this member, the biological function of gastric acid not only begins the process of digestion, but also the first line of defense against orals germs [5].

Traditional medicine had been important in treatment of disease since long ago and recently is gaining importance in treatment of diseases [5]. The doctrine of Iranian traditional medicine has strong arguments based on the use of experience and analogy based on the normal functioning of the body to understand and explain many symptoms and signs [6]. Particularly, the gastrointestinal tract is very important in traditional medicine, because when it is normal it is beneficial to the entire body, and if the stomach is not healthy, it can be harmed to the entire body [7].
In Iranian traditional medicine, the primary site of the stomach and the end of the esophagus is known as a sensitive area of the stomach. Due to the excessive sense of this area, stomach symptoms are generated more common in this area, but the sensation of pain can also be felt in the region of the heart or the left spleen. Stomach pain is mainly due to several factors [7].

The causes of stomachache in Iranian traditional medicine are as follows:

a) Pain with burning in stomach implies an accumulation of abnormal humeric substance in the stomach $[8,9]$.

b) "humour" which is called in the Iranian traditional medicine books" khelt" is a wet and fluid substance, normally there are four humours in the human body, Phlegm or Balgham, Blood or Dam, Yellow bile or Safra and Black bile or Sauda [10].

c) Post-meal pain is due to stimulation of Yellow bile or Safra and Black bile or Sauda in the stomach $[6,8,11]$.

d) Sometimes pain is due to excessive stretching caused by gas in the stomach [9], in this case, in addition to causing stomach pain, the patient is suffering from bloating, feeling stretching under the ribs. The cause of gas in the stomach can be due to the consumption of nourishing foods $[7,8]$. 
e) If accompanied with feeling heavy in the stomach, there is evidence of excessive filling of the stomach.

f) Other causes of stomach pain in Iranian traditional medicine are overeating, especially when hunger is not caused by stomach upset due to stomach upset in the stomach. In some cases, cold water also causes pain in these patients [7].

g) Stomach weakness is also a cause of stomach pain, which is caused by post-meal pain, which is due to the inability of the stomach to digest food, this condition only improves with vomiting or diarrhea $[7,8,11]$.

h) Sometimes stomachache is caused due to the type of food (hot foods likes pepper)(8).

i) The cause of gastric pain sometimes occurs due to excessive sensation of the stomach, which is caused by the least pain trigger(8).

Diagnosis and treatment of stomach pain is important, gastrointestinal tract disorders spreads to other organs and causes significant problems. Diseases in the of stomach may cause serious damage to the heart and liver and ultimately lead to death [7].

Therefore, we need to deal more seriously with signs and symptoms to diagnose it as soon as possible so that it can be prevented by treatment faster.

\section{References}

1. Hatami K, Pourshams A (2003) Dyspepsia, gastroesophageal reflux disease and irritable bowel syndrom among blood doners. Govaresh 8(4): 138-146.

2. Sayehmiri koroush, Tavan H (2016) Systematic review and metaanalysis methods prevalance of peptic ulcer in iran. govaresh 20(4): 250-258.

3. Hunt RH, Camilleri M, Crowe S (2015) The stomach in health and desease. Recent Adv Clin Pract 1-19.

4. Alizadeh M, Khadem E, Aliasl J (2017) Diagnosis protocol of stomach distemperament for clinical practice in iranian traditional medicine:a narrative review. Iran J Public Health (46): 877-81.

5. Sheikh Roshandel HR, Ghadimi F, Sheikh Roshandel R (2016) Developing and standardization of a structured questionnaire to determine the temperament (Mizaj) of individuals. Indian J Tradit Knowl 15(2): 341-346.

6. Babaeian M, Borhani M (2012) Gastrointestinal system in the viewpoint of traditional iranian medicine. J Islam Iran Tradit Med 2(4): 303-314.

7. Hajiheidari M, Babaeian M, Mazaheri M (2012) A review on causes of gastric pain in iranian traditional medicine. J Islam Iran Tradit Med.

8. Arzani M (2008) Teb-e-Akbari (Akbari,s medicine), ehya teb e tabiee, Tehran.

9. Borhani M, Shirzad M (2012) Semiology of gastric disease in traditional iranian medicine. J Islam Iran Tradit Med 2(4): 315-328.

10. Emtiazy M, Keshavarz M, Khodadoost M, Kamalinejad M, Gooshahgir et al. (2012) Relation between Body Humors and Hypercholesterolemia: An Iranian Traditional Medicine Perspective Based on the Teaching of Avicenna. Iran red crescent 14: 133-138.

11. Azam khan M (1869) Exir Azam (great elixir).

\section{Your next submission with Juniper Publishers will reach you the below assets}

Commons Attribution 4.0 License DOI: 10.19080/JCMAH.2018.08.555734 